\title{
Assessment of microbiota diversity in dental unit waterline contamination
}

\author{
Yun Dang ${ }^{\text {Equal first author, 1 }}$, Qian zhang ${ }^{\text {Equal first author, } 2}{ }^{2}$, Jing Wang ${ }^{3}$, Qian Wang ${ }^{1}$, Meng Han $^{4}$, Yuting Niu ${ }^{5}$, Hua Li ${ }^{\text {Corresp., } 1}$, \\ Xiue Li ${ }^{\text {Corresp. } 1}$ \\ 1 Department of Nursing, School of Stomatology, Peking University, Beijing, 010, \\ 2 Central Laboratory, School of Stomatology, Peking University, Beijing, 010, 10 \\ 3 Department of Preventive Dentistry, School of Stomatology, Peking University, Beijing, 010, 0 \\ ${ }^{4}$ The Fourth Outpatient Department, School of Stomatology, Peking University, Beijing, 010, \\ 5 Department of Pediatric Dentistry, School of Stomatology, Peking University, Beijing, 010, 0 \\ Corresponding Authors: Hua Li, Xiue Li \\ Email address: lihua770120@163.com, lixiue1216@163.com
}

Background. Dental unit waterlines (DUWLs) provide water for handpieces, air/water syringes, and mouth-rinse water outlets. DUWL contamination can negatively affect the operating environment and public health. So it is important to elucidate the bacterial concentrations and microbial composition in the DUWLs from different dental specialties. Methods. We collected 350 5-mL dental water samples (from high-speed handpieces, air/water syringes, and mouth-rinse water outlets) from 60 dental chair units (DCUs) at a dental hospital to determine the bacterial concentrations by culture methods. Meanwhile, to investigate the diversity and community structure of microbe in the DUWLs, 17 highquality DNA from 60 250-mL air/water syringe water samples, which were collected from the same 60 DCUs, were analyzed using 16S rDNA high-throughput sequencing. Results. The median bacterial concentration was $166(31.5,672.5) \mathrm{CFU} / \mathrm{mL}$ and the range was $0-3,816,000 \mathrm{CFU} / \mathrm{mL}$. Only $42.6 \%$ of the water samples had bacterial concentrations below $100 \mathrm{CFU} / \mathrm{mL}$. The Kruskal-Wallis $\mathrm{H}$-test revealed that the water samples from three dental specialties had significantly different bacterial concentrations $(H=27.441, P \square 0.01)$. Highthroughput sequencing results showed significant differences in bacterial community structure between periodontics and the other two departments. In the samples from three dental specialties, 508 OTUs were detected, with 160, 182 and 176 OTUs unique to the periodontics, endodontics and prosthodontics specialties, respectively. Linear discriminant analysis (LDA) effect size (LEfSe) suggested that Hydrocarboniphaga, Zoogloea, Aquabacterium, and Hydrogenophaga were enriched in the periodontics specialty; Acinetobacter, Geothrix, and Desulfovibrio were enriched in the prosthodontics specialty; and Alistipes, Clostridium XIVa, and Serratia were enriched in the endodontics specialty. Seven potentially human-pathogenic genera (Pseudomonas, Acinetobacter, 
Sphingomonas, Ochrobactrum, Rhizobium, Brevundimonas, and Methylobacterium) with relative abundance exceeding $1 \%$ were also detected in the DUWLS. Conclusions. The bacterial concentrations and microbial composition were influenced by different dental specialties, so a validated disinfection protocol should be used to control DUWL contamination in different dental specialties. 


\section{Assessment of microbiota diversity in dental unit waterline contamination}

2 Yun Dang ${ }^{1 *}$, Qian Zhang ${ }^{2 *}$, Jing Wang ${ }^{3}$, Qian Wang ${ }^{1}$, Meng Han ${ }^{4}$, Yuting Niu ${ }^{5}, \mathrm{Hua} \mathrm{Li}^{1{ }^{1 \sharp}}$ and Xiue $3 \mathrm{Li}^{1 \#}$

$5 \quad{ }^{1}$ Department of Nursing, School of Stomatology, Peking University, Beijing, China

$6 \quad{ }^{2}$ Central Laboratory, School of Stomatology, Peking University, Beijing, China;

$7 \quad{ }^{3}$ Department of Preventive Dentistry, School of Stomatology, Peking University, Beijing, China

$8{ }^{4}$ The Fourth Outpatient Department, School of Stomatology, Peking University, Beijing, China

$9 \quad{ }^{5}$ Department of Pediatric Dentistry, School of Stomatology, Peking University, Beijing, China

\section{Corresponding author}

$12{ }^{\#} \mathbf{H u a}$ Li: 22nd South Street of Zhongguancun, Haidian District, Beijing, 100081, China

13 Email address: lihua770120@163.com

14 \#Xiue Li: 22nd South Street of Zhongguancun, Haidian District, Beijing, 100081, China

15 Email address: lixiue1216@163.com

16

17 *Yun Dang and Qian Zhang contributed equally to this work. 


\section{Abstract}

24 Background. Dental unit waterlines (DUWLs) provide water for handpieces, air/water syringes, and mouth-rinse water outlets. DUWL contamination can negatively affect the operating environment and public health. So it is important to elucidate the bacterial concentrations and microbial composition in the DUWLs from different dental specialties.

Methods. We collected 350 5-mL dental water samples (from high-speed handpieces, air/water syringes, and mouth-rinse water outlets) from 60 dental chair units (DCUs) at a dental hospital to determine the bacterial concentrations by culture methods. Meanwhile, to investigate the diversity and community structure of microbe in the DUWLs, 17 high-quality DNA from 60 250-mL air/water syringe water samples, which were collected from the same 60 DCUs, were analyzed using 16S rDNA high-throughput sequencing.

34 Results. The median bacterial concentration was $166(31.5,672.5) \mathrm{CFU} / \mathrm{mL}$ and the range was 0 3,816,000 CFU/mL. Only $42.6 \%$ of the water samples had bacterial concentrations below 100 CFU/mL. The Kruskal-Wallis $H$-test revealed that the water samples from three dental specialties

37 had significantly different bacterial concentrations $(H=27.441, P<0.01)$. High-throughput sequencing results showed significant differences in bacterial community structure between periodontics and the other two specialties. In the samples from three dental specialties, 508 OTUs were detected, with 160, 182 and 176 OTUs unique to the periodontics, endodontics and prosthodontics specialties, respectively. Linear discriminant analysis (LDA) effect size (LEfSe) suggested that Hydrocarboniphaga, Zoogloea, Aquabacterium, and Hydrogenophaga were enriched in the periodontics specialty; Acinetobacter, Geothrix, and Desulfovibrio were enriched in the prosthodontics specialty; and Alistipes, Clostridium XIVa, and Serratia were enriched in the endodontics specialty. Seven potentially human-pathogenic genera (Pseudomonas, Acinetobacter, 
46 Sphingomonas, Ochrobactrum, Rhizobium, Brevundimonas, and Methylobacterium) with relative

47 abundance exceeding $1 \%$ were also detected in the DUWLs.

48 Conclusions. The bacterial concentrations and microbial composition were influenced by different

49 dental specialties, so a validated disinfection protocol should be used to control DUWL

50 contamination in different dental specialties.

52 Keywords

53 Dental unit waterlines, High-throughput sequencing, Microbiota, Water quality

\section{Introduction}

Dental unit waterlines (DUWLs) are vital components of dental chair units (DCUs) that provide water for handpieces, air/water syringes, and mouth-rinse water outlets (Hoogenkamp et al., 2020).

In recent years, iatrogenic infection caused by DUWL contamination has attracted much attention

(Noopan et al., 2019; Okubo et al., 2020). The first reported case of Legionella from DUWLs was that of an 82-year-old woman who died from Legionnaires' disease. L. pneumophila serogroup 1 was isolated from dental water samples and her bronchial aspirate (Ricci et al., 2012). Additionally, in 2015, nine children were infected by Mycobacterium abscessus after having a pulpotomy at a pediatric dentistry practice in Georgia, United States (Peralta et al., 2016). Microorganisms in the DUWLs, especially opportunistic pathogens, are potential risk factors for medically compromised or immunocompromised patients during dental care (Pankhurst, Scully \& Samaranayake, 2017). Consequently, it is important to prevent DUWL contamination. In some studies, the bacterial concentrations far exceeded the safety standards 
69 units $[\mathrm{CFU}] / \mathrm{mL})$ (Ji et al., 2016; Watanabe et al., 2016) and European Council (100 CFU/mL)

70 (Hoogenkamp et al., 2021; Ditommaso et al., 2016). Microorganisms in the DUWLs may come

71 from the supply water or patients' oral cavities via reverse suction (Volgenant \& Persoon, 2019).

72 These microorganisms can adhere to the tube walls and colonize to form biofilms in the complex

73 networks of thin tubes due to long periods of water stagnation (Hoogenkamp et al., 2020).

74 Established biofilms play important roles in continued DUWL contamination by releasing

75 fragments containing bacterial cell aggregates, and act as reservoirs of opportunistic pathogens by

76 protecting them from antimicrobials (Dahlen, 2021). Therefore, assessing bacterial contamination

77 and taking measures are necessary for public health.

DUWL contamination is primarily identified by bacterial culture and polymerase chain reaction (PCR) methods. However, culture may underestimate bacterial concentrations, because some bacteria exist in a viable but non-cultivable state in the environment (Hugenholtz, Goebel \&

81 Pace, 1998). Some opportunistic pathogens in the DUWLs are difficult to culture, while 82 polymerase chain reaction methods can be used only for identifying known strains, such as 83 Legionella spp. and Pseudomonas aeruginosa (Ditommaso et al., 2016; Ditommaso et al., 2019).

84 All the two traditional methods are insufficient to determine the actual microbial diversity and community structure in the DUWLs (Zhang et al., 2018). Thus, risk of cross-infection by unknown bacteria in the DUWLs might be ignored. The bacterial 16S rDNA high-throughput sequencing 87 platform can achieve the goal, with many advantages such as a fast sequencing speed, high accuracy, higher and entirety sequencing depth (Federici \& Soddu, 2020). Also, some studies have

89 applied high-throughput sequencing to elucidate microorganism diversity and community 90 structure in freshwater system and river water (Xiong \& Zhan, 2018; Zhou et al., 2021). 
Therefore, this study investigated the bacterial concentrations, diversity, and community

92 structure in the DUWLs from three dental specialties (periodontics, endodontics, prosthodontics)

93 using culture and high-throughput sequencing technology. The aim was to clarify the risks of

94 dental water and provide a basis for controlling contamination and establishing standards for 95 DUWLs.

97 Materials and Methods

98 Water sample collection

99 Water samples were collected from 60 DCUs at a dental hospital: 19, 21, and 20 DCUs in the

100 periodontics (PE-M), endodontics (EN-M), and prosthodontics (PR-M) specialties, respectively

101 (Table S1). All of the DCUs were used for standard dental treatments, except those in the operating

102 room. The basic information of DCUs in three dental specialties was as follows: average operating

103 years (PE-M: 7, EN-M: 10, PR-M: 12), average patients per month (PE-M: 139, EN-M: 156, PR-

104 M: 161) and average patients per day of DCU (PE-M: 6, EN-M: 6, PR-M: 7). The DUWLs were

105 supplied with municipal water that meets the national standards of China, i.e., "Standard

106 Examination Methods for Drinking Water-Microbiological Parameters£ (GB/T 5750.12-2006)

107 (heterotrophic plate count $<100 \mathrm{CFU} / \mathrm{mL}$ at $37^{\circ} \mathrm{C}$ ).

108 For cultivation: Before sampling, the dental water outlets were disinfected with alcohol-

109 soaked cotton balls, and the waterlines were flushed for 2 min before daily dental practice and 30

$110 \mathrm{~s}$ after daily dental practice. $5 \mathrm{~mL}$ water samples were collected separately into sterilized tubes

111 from the high-speed handpieces, air/water syringes, and mouth-rinse water outlets before and after

112 daily dental practice $(14,21$, and 20 DCUs in the periodontics, endodontics, and prosthodontics

113 specialties, respectively). In addition, $5 \mathrm{~mL}$ water samples were collected only from the air/water 
114 syringes and mouth-rinse water outlets before and after daily dental practice from the other five

115 DCUs in the periodontics specialty, because the high-speed handpieces of these DCUs were not in 116 use.

For high-throughput sequencing: $250 \mathrm{~mL}$ water samples from air/water syringes were

118 collected into sterilized bottles from all 60 DCUs at the same time with the $5 \mathrm{~mL}$ water samples

119 collecting before daily dental practice.

120 Bacterial culture

121 The $5 \mathrm{~mL}$ water samples were diluted to $1: 10$ and 1:100 using purified water. Then, $500 \mu \mathrm{L}$ of

122 each diluted solution was cultivated on a brain heart infusion (BHI) plate (BD, Franklin Lakes, NJ,

123 USA). The plates were incubated for 48 hours at $37^{\circ} \mathrm{C}$ in an incubator containing $5 \% \mathrm{CO}_{2}$. The

124 viable bacterial counts were calculated as CFU/mL. According to China's national standards, 100

$125 \mathrm{CFU} / \mathrm{mL}$ was used as the threshold value.

\section{Statistical analysis of Bacterial concentrations}

127 Kolmogorov-Smirnov test was performed to test for normal distribution on all data. The results

128 showed that the bacterial concentrations of three dental specialties were non-normally distributed

129 data $(P<0.01)$. The Median $(\mathrm{Q} 25, \mathrm{Q} 75)$ of bacterial concentrations were calculated for descriptive

130 analysis. The Wilcoxon signed-rank test was used to compare bacterial concentrations before and

131 after daily dental practice. The Kruskal-Wallis $H$-test was used to assess the differences in

132 bacterial concentrations among the endodontics, periodontics, and prosthodontics specialties. $P$ -

133 values were two-sided and a $P$-value $<0.05$ was considered to indicate statistical significance.

\section{DNA extraction}

135 The $250 \mathrm{~mL}$ water samples from air/water syringes of 60 DCUs before daily dental practice were

136 filtered through a $0.2-\mu \mathrm{m}$ pore polycarbonate filter (Millipore, Billerica, MA, USA), which was 
137 then stored in phosphate-buffered saline at $4^{\circ} \mathrm{C}$. Microorganism precipitates were obtained by

138 centrifugation at $12,000 \mathrm{rpm}$ for $10 \mathrm{~min}$; the filter membrane was discarded. Bacterial genomic

139 DNA was extracted using a Takara MiniBEST Bacterial Genomic DNA Extraction Kit (Takara,

140 Dalian, China), following the manufacturer's protocol. High-quality DNA with $\mathrm{OD}_{260 / 280}=1.8-$

1412.0 , concentration $>5 \mathrm{ng} / \mu \mathrm{L}$, and no degradation with agarose gel electrophoresis (from 17

$142 \mathrm{air} /$ water syringe water samples) was stored at $-80^{\circ} \mathrm{C}$ until being used for molecular applications.

143 High-throughput sequencing

144 The high-quality extracted DNA was used for further PCR amplification with primers targeting

145 the V3-V4 hypervariable regions of bacterial 16S rRNA genes. The primers were 314F (5-

146 CCTACGGGRSGCAGCAG-3) and 806R (5-GGACTACVVGGGTATCTAATC-3) with an 8-bp

147 unique barcode. PCR amplification was performed using a KAPA HiFi HotStart ReadyMix PCR

148 kit (Roche, Basel, Switzerland) using a $20 \mu \mathrm{L}$ reaction mixture. Thermal cycling conditions were

149 as follows: an initial denaturation at $95^{\circ} \mathrm{C}$ for $3 \mathrm{~min}, 27$ cycles of $95^{\circ} \mathrm{C}$ for $30 \mathrm{~s}, 55^{\circ} \mathrm{C}$ for $30 \mathrm{~s}$, and

$15072^{\circ} \mathrm{C}$ for $45 \mathrm{~s}$, and a final extension at $72^{\circ} \mathrm{C}$ for $10 \mathrm{~min}$. The PCR products were examined using

$1512 \%$ agarose gel electrophoresis and purified using an AxyPrep Gel Extraction kit (Axygen, Union

152 City, CA, USA). Equimolar samples were pooled together for sequencing using the Illumina

153 MiSeq PE250 platform (Shanghai Realgene Biotech, Shanghai, China). The raw sequence data

154 had been submitted to NCBI under Accession Number PRJNA690183.

155 Statistical analysis of sequencing data

156 The paired-end reads were merged based on the overlap base pairs using PANDAseq software

157 (Masella et al., 2012). Clean merged reads were obtained with an average quality $>20$ and base $\mathrm{N}$

$158<3$. The sequences were clustered into operational taxonomic units (OTUs) at a 97\% similarity

159 level using USEARCH after removing chimeras and singletons (Edgar, 2013). Based on optimized 
160 OTUs, Alpha-diversity indices (including Chao1, Observed species, Shannon, and Simpson

161 indices) were calculated by extracting the same reads from each sample and compared using the

162 Kruskal-Wallis $H$-test to determine the significance of differences in bacterial diversity among the

163 dental specialties. The taxonomic annotations (phylum, class, order, family, and genus) of OTUs

164 were identified using the RDP database. Principal coordinates analysis (PCoA) and hierarchical

165 cluster analysis were performed with the nonparametric analysis of variance using distance

166 matrices (ADONIS) to examine the bacterial communities in three dental specialties.

\section{Results}

169 Microbial culture of water samples

170 A total of 350 dental water samples were collected. The median bacterial concentration was 166

$171(31.5,672.5) \mathrm{CFU} / \mathrm{mL}$ and the range was $0-3,816,000 \mathrm{CFU} / \mathrm{mL}$. Only $42.6 \%$ of the water samples

172 had bacterial concentrations below the threshold of China's national drinking-water standards

173 (heterotrophic plate counts $<100 \mathrm{CFU} / \mathrm{mL}$ ).

174 Differences in bacterial concentrations among the samples from the periodontics,

175 endodontics, and prosthodontics specialties were significant (Table 1). There was no significant

176 difference in bacterial concentrations between samples before and after daily dental practice (Table

177 2).

178 Overview of sequencing results

179 After quality control and filtering, 603,296 clean reads were obtained from 17 dental water samples

180 (6, 5, and 6 samples from the periodontics, endodontics, and prosthodontics specialties,

181 respectively) with an average of $35,488 \pm 1,173$ sequences per sample (range: $33,120-37,988$ ),

182 representing 7,130 OTUs. On average, 419 OTUs were detected in each sample (range: 262-562). 
183 Bacteria composition in the periodontics, endodontics, and prosthodontics specialties

184 The main phylum, with a relative abundance more than $85 \%$, was Proteobacteria (Fig. $1 A$ ). Other

185 phyla included Bacteroidetes, Acidobacteria, Chloroflexi, and Firmicutes. Twenty genera were

186 detected in the 17 water samples from the periodontics (PE-M), endodontics (EN-M), and

187 prosthodontics (PR-M) specialties. Seven potentially human-pathogenic genera with relative

188 abundances exceeding 1\% were detected in the 17 samples from three dental specialties, including

189 Pseudomonas (relative abundance: $31.08 \%)$, Acinetobacter $(7.64 \% ; 21.05 \%$ for PR-M, $<1 \%$ for

190 PE-M and EN-M), Sphingomonas (2.68\%), Ochrobactrum (2.71\%), Rhizobium (1.47\%; 2.61\%

191 for PE-M, 1.04\% for PR-M, < 1\% for EN-M), Brevundimonas (1.05\%; 1.13\% for PE-M, 1.26\%

192 for PR-M, < 1\% for EN-M), and Methylobacterium (1.88\%; 5.48\% for EN-M, < 1\% for PE-M and

193 PR-M) (Fig. 1B). Other genera included Sphingobium (9.61\%), Curvibacter (10.93\%),

194 Acidovorax (5.95\%), Hydrogenophaga (3.00\%), Hydrocarboniphaga (2.10\%), Zoogloea (1.71\%),

195 and Aquabacterium (1.22\%). Hydrocarboniphaga was detected only in the periodontics specialty,

196 while Zoogloea and Aquabacterium were detected in the periodontics and prosthodontics

197 specialties.

198 Bacterial diversity in the periodontics, endodontics, and prosthodontics specialties

199 The alpha-diversity indices, which estimated bacteria richness (Chao1 and observed species

200 indices) and diversity (Shannon and Simpson indices), are presented in Table 3. There were no

201 significant differences in diversity indices among the periodontics, endodontics, and

202 prosthodontics specialties $(P>0.05)$. However, PCoA and ADONIS showed significant

203 differences in bacterial community structure among the 17 samples from three dental specialties

204 (Fig. 2). Samples from the endodontics and prosthodontics specialty clustered together, whereas 
205 samples from the periodontics specialty were far removed from the other two, indicating differing

206 community structures among the different dental specialties.

207 Different bacterial communities in the periodontics, endodontics, and prosthodontics specialties

208 There were 860, 945, and 963 bacterial OTUs in the periodontics, endodontics, and prosthodontics

209 specialties, respectively, and 508 OTUs were detected in all samples of the three specialties (Fig.

$2103 A$ ). The OTUs with detection frequencies exceeding 50\% were showed in Fig. 3B. There were

211160,182 , and 176 OTUs unique to the periodontics, endodontics, and prosthodontics specialties,

212 respectively. OTUs unique to the periodontics specialty included Sediminibacterium, Solimonas,

213 Legionella, Bdellovibrio, Hydrocarboniphaga, Flavobacterium, Cupriavidus, and Azospira; those

214 unique to the endodontics specialty included Gp16, Parcubacteria genera incertae sedis, and

215 Saccharibacteria genera incertae sedis; only Gp3 was unique to the prosthodontics specialty. The

216 periodontics specialty shared 592 and 716 OTUs with the endodontics and prosthodontics

217 specialties, respectively, while the endodontics and prosthodontics specialties shared 679 OTUs 218 (Fig. 3A).

219 Linear discriminant analysis (LDA) effect size (LEfSe) was used to identify the presence 220 and effect size of region-specific OTUs among the water samples of three specialties (Fig. 4). The 221 results suggested that Acinetobacter, Gp17, Geothrix, and Desulfovibrio were enriched in the 222 prosthodontics specialty; Alistipes, Clostridium XIVa, and Serratia were enriched in the 223 endodontics specialty; and Hydrocarboniphaga, Zoogloea, Aquabacterium, and Hydrogenophaga 224 were enriched in the periodontics specialty. 


\section{Discussion}

227 DUWL Contamination puts patients at potential risk during dental care. Therefore, this study

228 analyzed the bacterial concentrations, diversity, and community structure in DUWLs of

229 periodontics, endodontics, and prosthodontics specialties to provide a basis for controlling

230 contamination and establishing standards for DUWLs.

231 Bacteria can be easily colonized in the DUWLs environment. Previous studies had showed

232 that the bacterial concentrations can reached $1.4 \times 10^{5}$ and $1.8 \times 10^{6} \mathrm{CFU} / \mathrm{mL}$ (Watanabe et al.,

233 2016; Ji et al., 2018). Also in our study, bacterial concentrations ranged from 0 to 3,816,000

$234 \mathrm{CFU} / \mathrm{mL}$, and only $42.6 \%$ of water samples met China's national drinking-water standards. And

235 the bacterial concentrations of water samples differed significantly in three specialties. We also

236 compared water samples before and after daily dental practice and found no significant difference

237 in bacterial concentrations. The probable cause of the result was that although flushing during

238 dental care could reduce bacterial concentrations to an extent (Watanabe et al., 2008), suck-back

239 of saliva and blood still made contamination a serious concern (Spagnolo, Sartini \& Cristina,

240 2020). Some main factors of DUWL contamination were the small lumen size $(0.5-2 \mathrm{~mm})$ and

241 materials of tubes, high surface-to-volume ratio (6:1), slow water flow during working hours and

242 water stagnation outside of working hours (Spagnolo, Sartini \& Cristina, 2020). These factors

243 allowed bacteria to be easily colonized in the DUWLs environment and to contaminate output

244 water. If dental water was not appropriately treated, patients' health could be at risk.

245 In our study, Proteobacteria was a prevalent phylum in DUWLs with the relative

246 abundance over $85 \%$ of the total phyla, which was similar with previous studies which showed

247 that Proteobacteria was also the most abundant phylum in biofilm samples (Fan et al., 2021) and

248 in dental water (Costa et al., 2015; Yoon \& Lee, 2019). Meanwhile, because of the high tolerance 
249 to chlorine compared with other phylum, Proteobacteria was also the most commonly detected

250 phylum in water distribution systems (Huang et al. 2014; Holinger et al., 2014). The predominant

251 bacterial genera with relative abundance more than 5\% detected in our study were Pseudomonas,

252 Curvibacter, Sphingobium, Acinetobacter and Acidovorax. Among these five genera,

253 Pseudomonas and Acinetobacter were human-pathogenic bacteria, which were also detected in

254 other researches (Costa et al., 2015; Zhang et al., 2018; Yoon \& Lee, 2019; Fan et al., 2021).

255 Pseudomonas could colonize and form biofilm in plastic waterlines (Spagnolo, Sartini \& Cristina,

256 2020). Pseudomonas spp., especially Pseudomonas aeruginosa, could grow in low-nutrient

257 environment and often exhibit resistance to antimicrobial agents and disinfectants (Rodrigues et

258 al., 2017). Acinetobacter was also a well-known cause of nosocomial infections, and exhibited a

259 high degree of drug resistance (Harding, Hennon \& Feldman, 2018). These pathogens may put

260 medically compromised or immunocompromised individuals at risk of cross-infection during

261 dental care. Besides, endotoxins released from gram-negative pathogens in the DUWLs might

262 introduce allergic airway reaction (Coleman et al., 2009) and increase the release of

263 proinflammatory cytokines in gingival tissue during oral surgery (Putnins, Di Giovanni \& Bhullar,

264 2001). Therefore, a disinfection protocol should be used to reduce DUWL contamination.

265 Chemical disinfectants, such as peracetic acid, hydrogen peroxide, sodium hypochlorite, chlorine

266 dioxide, chlorhexidine, 2,100 ppm ozone, iodine cartridges, and super-oxidized water, can be used

267 either periodically or continuously to treat DUWL biofilms (Spagnolo, Sartini \& Cristina, 2020;

268 Cicciu, 2020). Physical measures, including filtration, flushing, and reverse osmosis, should also

269 be combined with disinfection measures. In addition, the scheduled technical maintenance of

270 dental units and monitoring the quality of dental water are equally important. The pathogenic 
271 mechanisms, independent factors, and drug resistance of pathogenic bacteria should also be

272 explored further to provide a basis for DUWL disinfection.

273 Dental practices and treated patients might be important factors for bacterial contamination

274 in different dental specialties. Patients with different oral diseases are treated in different dental

275 specialties with different dental practices. The oral microbiome is the driving factor of oral

276 diseases. For example, Porphyromonas gingivalis, which is the main pathogen responsible for

277 periodontitis, and Streptococcus mutans, which gives rise to dental caries (He et al., 2015), may

278 enter DUWLs in the periodontics and endodontics specialties via suck-back. As stated before, the

279 bacterial concentrations of water samples differed significantly in three specialties of our study.

280 The periodontics specialty had a significantly different DUWL bacterial community structure from

281 the endodontics and prosthodontics specialties and harbored more genera that were not found in

282 other two specialties. Additionally, the relative abundance of four pathogenic genera

283 (Acinetobacter, Rhizobium, Brevundimonas and Methylobacterium) also differed among the three

284 dental specialties. The results were consistent with Zhang et al. (2018), which reported that the

285 water quality in an endodontics specialty was better than that in a periodontics specialty. Yoon \&

286 Lee (2019) also found similar bacterial diversities in the dental water of pedodontics and

287 periodontics specialties, while the dental water of a private dental clinic had distinct bacterial

288 diversity. It prompted that bacteria may have different distributions in water samples among the

289 dental specialties, possibly because of the suck-back of saliva and blood during dental care.

290 Although oral microorganisms have lower survival in the DUWLs than oral cavity, the

291 colonization and distribution of other water microorganisms in the DUWLs may still be affected

292 by short-term interactions with oral microorganisms. In addition, differences in hydrodynamic

293 stress among the three specialties may also affect the variation in microbial communities and 
294 bacterial concentrations by impacting the composition, density, and structure of biofilms

295 (Hoogenkamp et al., 2020). Therefore, a validated disinfection protocol should be used to control

296 DUWL contamination in different specialties. In addition, anti-retraction valves are usually fitted

297 distally to handpieces and air/water syringes to prevent suck-back. However, Ji et al. (2016)

298 pointed that $51.72 \%$ of DCUs failed the retraction evaluation because of anti-retraction valve

299 failure after a few months' use. Dental staff should monitor the efficacy of anti-retraction valve

300 through a retraction measurement device and the volume of water retraction should not exceed 40

$301 \mu \mathrm{L}($ Ji et al., 2016).

302 Conclusions

303 Our study used culture and high-throughput sequencing to analyze the bacterial

304 concentrations, diversity, and community structure in the DUWLs. The bacterial concentrations

305 ranging from 0 to $3,816,000 \mathrm{CFU} / \mathrm{mL}$ revealed DUWL contamination required more attention.

306 Proteobacteria was the most dominant phylum in the DUWLs. Among the predominant bacterial

307 genera detected in our study, Pseudomonas and Acinetobacter were human-pathogenic bacteria.

308 Therefore, disinfection measures are required to control DUWL contamination. In addition, the

309 scheduled technical maintenance of dental units and monitoring the quality of dental water are

310 equally important. The bacterial concentrations of dental water samples differed significantly in

311 three specialties and the periodontics specialty had a significantly different DUWL bacterial

312 community structure from the endodontics and prosthodontics specialties, possibly because of the

313 short-term interactions with oral microorganisms due to suck-back and differences in

314 hydrodynamic stress among the three specialties. The results suggested that a validated

315 disinfection protocol should be used to control DUWL contamination in different specialties. 


\section{References}

318 Cicciu M. 2020. Water contamination risks at the dental clinic. Biology (Basel) 9(3): 43 DOI:

$319 \quad$ org/10.3390/biology9030043.

320 Coleman DC, O’Donnell MJ, Shore AC, Russell RJ. 2009. Biofilm problems in dental unit water

321 systems and its practical control. Journal of Applied Microbiology 106: 1424-1437 DOI:

$322 \quad 10.1111 /$ j.1365-2672.2008.04100.x.

323 Costa D, Mercier A, Gravouil K, Lesobre J, Delafont V, Bousseau A, Verdon J, Imbert C. 2015.

324 Pyrosequencing analysis of bacterial diversity in dental unit waterlines. Water Research 81:

325 223-231 DOI: 10.1016/j.watres.2015.05.065.

326 Dahlen G. 2021. Biofilms in dental unit water lines. Monographs in Oral Science 29: 12-18 DOI:

$327 \quad 10.1159 / 000510195$.

328 Ditommaso S, Giacomuzzi M, Ricciardi E, Memoli G, Zotti CM. 2019. Colonization by

329 Pseudomonas aeruginosa of dental unit waterlines and its relationship with other bacteria:

330 suggestions for microbiological monitoring. Journal of Water and Health 17(4): 532-539 DOI:

$331 \quad$ 10.2166/wh.2019.240.

332 Ditommaso S, Giacomuzzi M, Ricciardi E, Zotti CM. 2016. Cultural and molecular evidence of

333 Legionella spp. colonization in dental unit waterlines: Which is the best method for risk

334 assessment? International Journal of Environmental Research and Public Health 13(2): 211

335 DOI: $10.3390 /$ ijerph13020211.

336 Edgar R. 2013. UPARSE: highly accurate OTU sequences from microbial amplicon reads. Nature 337 methods 10: 996-8 DOI: 10.1038/nmeth.2604. 
338 Fan CC, Gu HJ, Liu LM, Zhu HW, Yan J, Huo YB. 2021. Distinct microbial community of 339 accumulated biofilm in dental unit waterlines of different specialties. Frontiers in Cellular and 340 Infection Microbiology 11: 670211 DOI: 10.3389/fcimb.2021.670211.

341 Federici G, Soddu S. 2020. Variants of uncertain significance in the era of high-throughput genome 342 sequencing: a lesson from breast and ovary cancers. Journal of Experimental \& Clinical 343 Cancer Research 39(1): 46 DOI: 10.1186/s13046-020-01554-6.

344 Harding CM, Hennon SW, Feldman MF. 2018. Uncovering the mechanisms of Acinetobacter baumannii virulence. Nature Reviews Microbiology 16: 91-102 DOI: 10.1038/nrmicro.2017.148.

347 He J, Li Y, Cao Y, Xue J, Zhou X. 2015. The oral microbiome diversity and its relation to human 348 diseases. Folia Microbiologica (Praha) 60(1): 69-80 DOI: 10.1007/s12223-014-0342-2.

349 Holinger EP, Ross KA, Robertson CE, Stevens MJ, Harris JK, Pace NR. 2014. Molecular analysis 350 of point-of-use municipal drinking water microbiology. Water Research 49: 225-235 DOI: 10.1016/j.watres.2013.11.027.

352 Hoogenkamp MA, Brandt BW, de Soet JJ, Crielaard W. 2020. An in-vitro dynamic flow model 353 for translational research into dental unit water system biofilms. Journal of Microbiological 354 Methods 171: 105879 DOI: 10.1016/j.mimet.2020.105879.

355 Hoogenkamp MA, Brandt BW, Laheij AMGA, de Soet JJ, Crielaard W. 2021. The microbiological 356 load and microbiome of the Dutch dental unit; 'please, hold your breath' . Water Research 200: 117205 DOI: 10.1016/j.watres.2021.117205.

Huang K, Zhang XX, Shi P, Wu B, Ren H. 2014. A comprehensive insight into bacterial virulence 359 in drinking water using 454 pyrosequencing and Illumina high-throughput sequencing. Ecotoxicology and Environmental Safety 109: 15-21 DOI: 10.1016/j.ecoenv.2014.07.029. 
361 Hugenholtz P, Goebel BM, Pace NR. 1998. Impact of culture-independent studies on the emerging

362 phylogenetic view of bacterial diversity. Journal of Bacteriology 180: 4765-4774 DOI:

$363 \quad 10.1128 / \mathrm{JB} \cdot 180.18 .4765-4774.1998$.

364 Ji XY, Fei CN, Zhang Y, Zhang W, Liu J, Dong J. 2016. Evaluation of bacterial contamination of

365 dental unit waterlines and use of a newly designed measurement device to assess retraction of

366 a dental chair unit. International Dental Journal 66(4): 208-214 DOI: 10.1111/idj.12225.

367 Ji XY, Fei CN, Zhang Y, Liu J, Liu H, Song J. 2018. Three key factors influencing the bacterial

368 contamination of dental unit waterlines: a 6-year survey from 2012 to 2017. International

369 Dental Journal 69(3): 192-199 DOI: 10.1111/idj.12456.

370 Masella A, Bartram A, Truszkowski J, Brown D, Neufeld J. 2012. PANDAseq: paired-end

371 assembler for Illumina sequences. BMC Bioinformatics 13: 31 DOI: 10.1186/1471-2105-13-

37231.

373 Noopan S, Unchui P, Techotinnakorn S, Ampornaramveth RS. 2019. Plasma sterilization

374 effectively reduces bacterial contamination in dental unit waterlines. International Journal of

375 Dentistry 2019: 5720204 DOI:10.1155/2019/5720204.

376 Okubo K, Ito T, Okamoto K, Yamamoto I, Mizutani H, Kawata Y, Shiota Y, Ito M, Nakamura S,

377 Tai M, Yamamoto T, Takashiba S. 2020. Evaluation of the simulator with automatic irrigation

378 control system designed for countermeasures of internal contamination in dental unit water

379 lines. Heliyon 6(6): e04132 DOI: 10.1016/j.heliyon .e04132.

380 Pankhurst C, Scully C, Samaranayake L. 2017. Dental Unit Water Lines and their Disinfection

381 and Management: A Review. Dental update 44: 284-292 DOI: 10.12968/denu.2017.44.4.284.

382 Peralta G, Tobin-D’Angelo M, Parham A, Edson L, Lorentzson L, Smith C, Drenzek C. 2016.

383 Mycobacterium abscessus infections among patients of a pediatric dentistry practice- 
384

385

386

387

388

389

390

391

392

393

394

395

396

397

398

399

400

401

402

403

404 405

406

Georgia, 2015. Morbidity and Mortality Weekly Report 65(13): 355-356 DOI: 10.15585/mmwr.mm6513a5.

Putnins EE, Di Giovanni D, Bhullar AS. 2001. Dental Unit waterline contamination and its possible implications during periodontal surgery. Journal of Periodontology 72: 393-400 DOI: 10.1902/jop.2001.72.3.393.

Ricci ML, Fontana S, Pinci F, Fiumana E, Pedna MF, Farolfi P, Sabattini MAB, Scaturro M. 2012. Pneumonia associated with a dental unit waterline. The Lancet 379(9816): 684 DOI: 10.1016/S0140-6736(12)60074-9.

Rodrigues S, Suvarna S, Suvarna J, Saralaya V, Saldanha S, Shenoy VK. 2017. Microbial assessment of dental unit waterlines in an institutional setup in Karnataka, South India. Indian Journal of Dental Research 28(5): 555-559 DOI: 10.4103/ijdr.IJDR_775_16.

Spagnolo AM, Sartini M, Cristina ML. 2020. Microbial contamination of dental unit waterlines and potential risk of infection: A narrative review. Pathogens 9(8): 651 DOI: 10.3390/pathogens 9080651 .

Volgenant CMC, Persoon IF. 2019. Microbial water quality management of dental unit waterlines at a dental school. Journal of Hospital Infection 103(1): e115-e117 DOI: 10.1111/j.20411626.2012.00135.x.

Watanabe A, Tamaki N, Yokota K, Matsuyama M, Kokeguchi S. 2016. Monitoring of bacterial contamination of dental unit water lines using adenosine triphosphate bioluminescence. Journal of Hospital Infection 94(4): 393-396 DOI: 10.1016/j.jhin.2016.08.001.

Watanabe E, Agostinho A, Matsumoto W, Ito I. 2008. Dental unit water: bacterial decontamination of old and new dental units by flushing water. International Journal of Dental Hygiene 6(1): 56-62 DOI: 10.1111/j.1601-5037.2007.00278.x. 
407 Xiong W, Zhan A. 2018. Testing clustering strategies for metabarcoding-based investigation of 408 community-environment interactions. Molecular Ecology Resources 18: 1326-1338, DOI: $409 \quad 10.1111 / 1755-0998.12922$.

410 Yoon H, Lee S. 2019. An assessment of the bacterial diversity found in dental unit waterlines using 411 the Illumina MiSeq. Biocontrol Science 24(4): 201-212 DOI: 10.4265/bio.24.201.

412 Zhang Y, Ping Y, Zhou R, Wang J, Zhang G. 2018. High throughput sequencing-based analysis 413 of microbial diversity in dental unit waterlines supports the importance of providing safe water 414 for clinical use. Journal of Infection and Public Health 11(3): 357-63 DOI: $415 \quad$ 10.1016/j.jiph.2017.09.017.

416 Zhou R, Wang Y, Hilal MG, Yu Q, Feng, T, Li H. 2021. Temporal succession of water 417 microbiomes and resistomes during carcass decomposition in a fish model. Journal of 418 Hazardous Materials 403: 123795 DOI: 10.1016/j.jhazmat.2020.123795. 


\section{Figure 1}

Dominant bacterial communities in 17 water samples from the periodontics (PE-M), endodontics (EN-M), and prosthodontics (PR-M) specialties.

(A) Dominant bacterial communities at the phylum level in different specialties. (B) Dominant bacterial communities at the genus level in different specialties. 

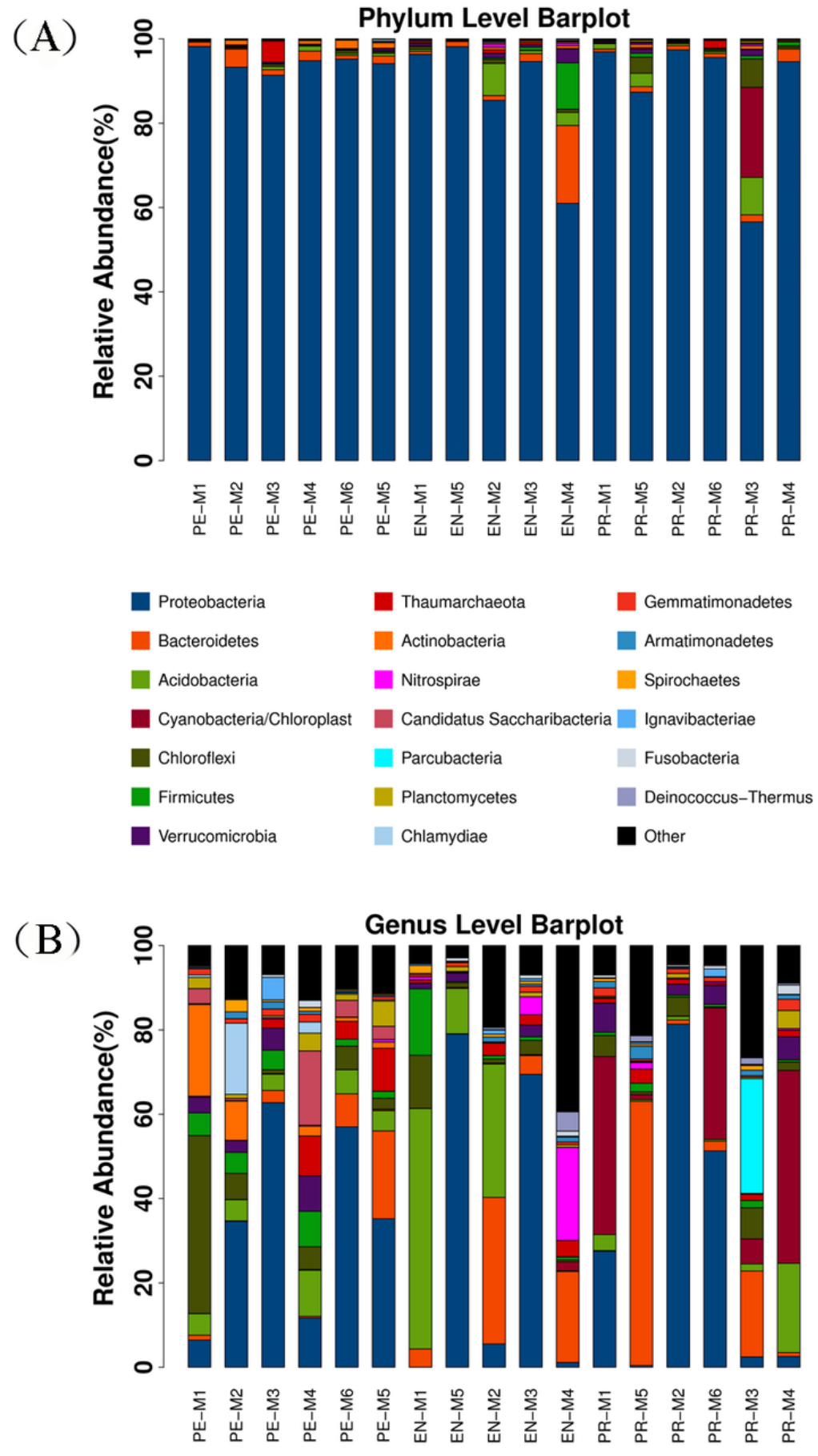

$\begin{array}{lll}\square \text { Pseudomonas } & \text { Sphingomonas } & \text { Brevundimonas } \\ \square \text { Curvibacter } & \text { Hydrocarboniphaga } & \text { Bradyrhizobium } \\ \square \text { Sphingobium } & \text { Methylobacterium } & \text { Sphingopyxis } \\ \square \text { Acinetobacter } & \text { Zoogloea } & \text { Nitrosopumilus } \\ \square \text { Acidovorax } & \text { Chlorophyta } & \text { Delftia } \\ \square \text { Hydrogenophaga } & \text { Rhizobium } & \text { Escherichia/Shigella } \\ \square \text { Ochrobactrum } & \text { Aquabacterium } & \text { Other }\end{array}$




\section{Figure 2}

Principal coordinates analysis (PCOA) and analysis of variance using distance matrices (ADONIS) of the 17 water samples from the periodontics (PE-M), endodontics (EN-M), and prosthodontics (PR-M) specialties.
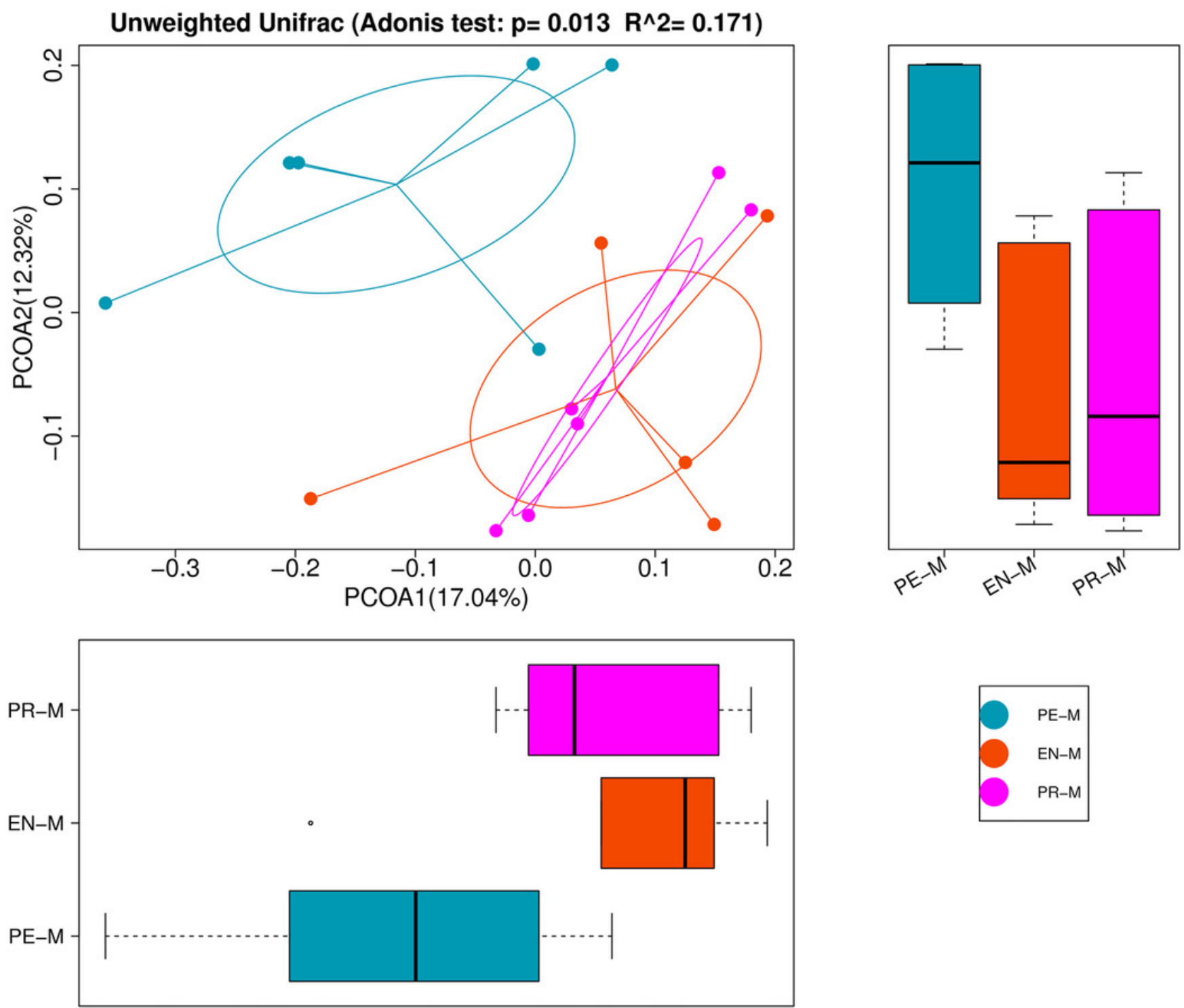


\section{Figure 3}

The shared and unique OTUs among the periodontics (PE-M), endodontics (EN-M), and prosthodontics (PR-M) specialties.

(A) Venn diagram of the numbers of shared and unique OTUs among the three specialties.

(B) The taxonomic nomenclature of shared and unique OTUs among the three specialties.

The OTUs in (B) were present in at least $50 \%$ of the samples and had a mean relative abundance of $>0.01 \%$ of all sequences. 
(A)

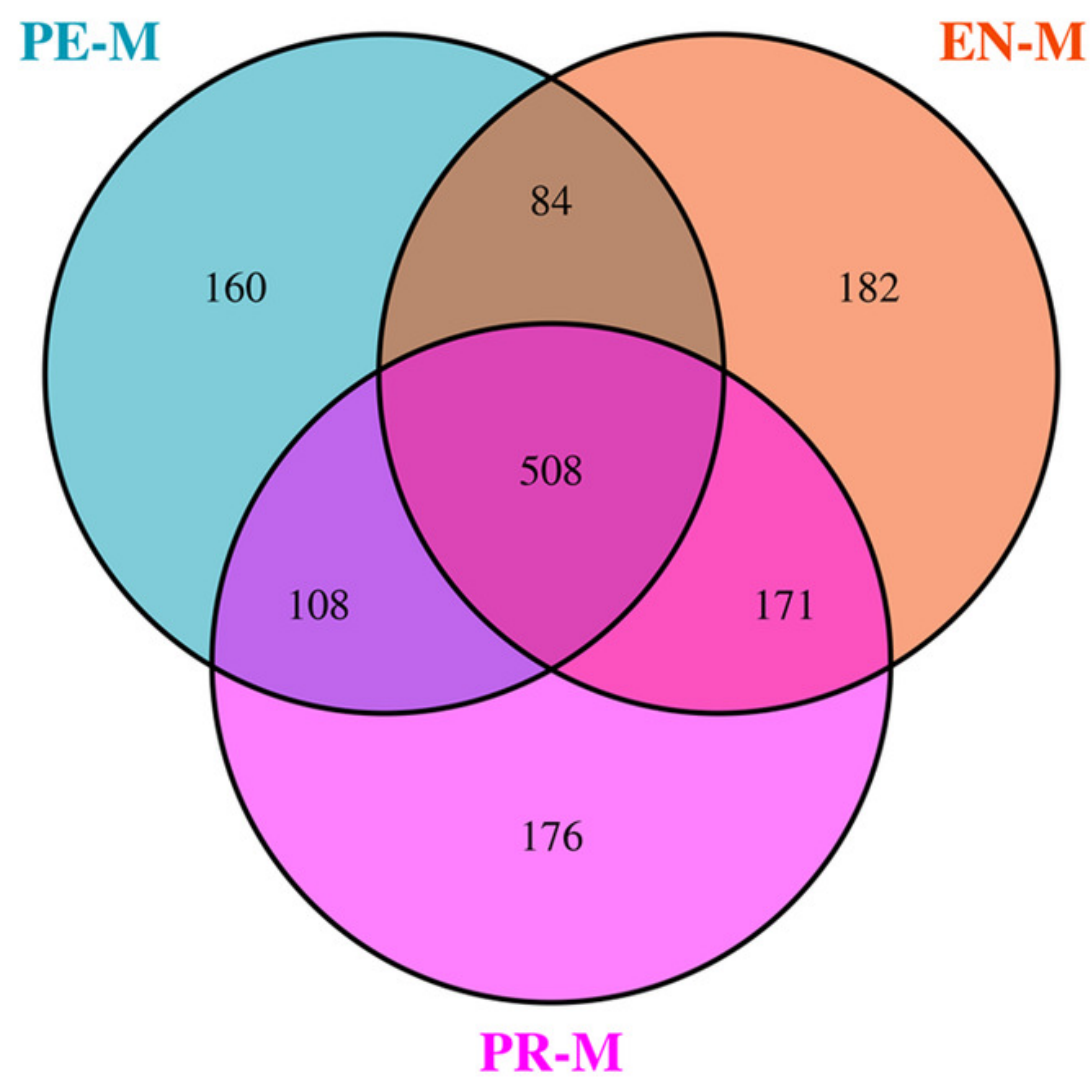

(B)

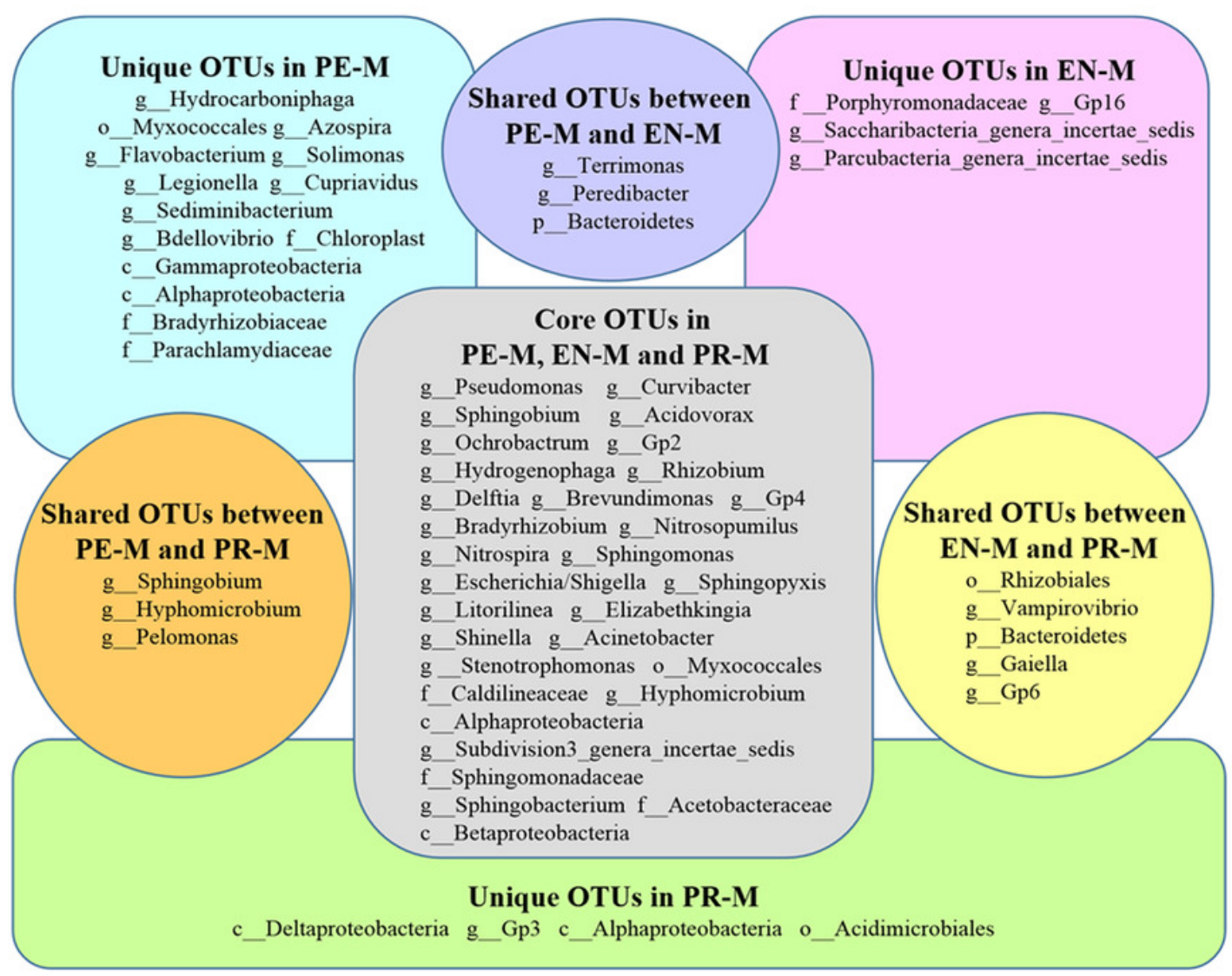


Figure 4

The distribution of microbial organisms in the periodontics (PE-M), endodontics (EN-M), and prosthodontics (PR-M) specialties.

(A) Cladogram analysis. (B) Linear discriminant analysis (LDA) effect size (LEfSe) 
(A)

$$
\begin{aligned}
& \square \text { PE-M } \\
& \square \text { EN-M } \\
& \square \text { PR-M }
\end{aligned}
$$

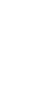

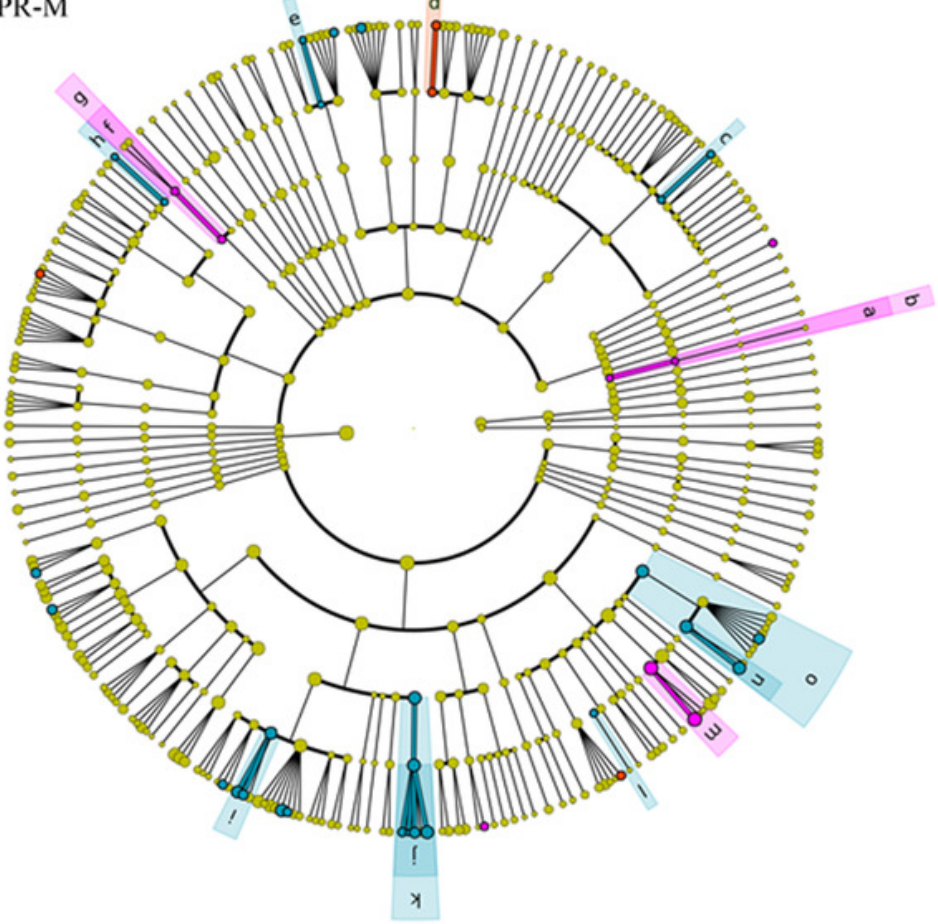

$\square$ a:g_Gpl7

b:c_Acidobacteria_Gp17

$\square$ c:f_Kineosporiaceae

$\square$ d:f Rikenellaceae

$\square$ e:f_Saprospiraceae

$\square$ f:f Thermaceae

g:o Thermales

$\square$ h:f_Bacillaceae

$\square$ i:f Burkholderiales incertae sedis

$\square$ j:f_Rhodocyclaceae

$\square$ k:o_Rhodocyclales

$\square \mathrm{l}: \mathrm{g}$ Solimonas

$\square$ m:f_Moraxellaceae

$\square$ n:f_Sinobacteraceae

o:0_Xanthomonadales

(B)

$\square \mathrm{PE}-\mathrm{M} \square \mathrm{EN}-\mathrm{M} \square \mathrm{PR}-\mathrm{M}$

f_Moraxellaceae g_Gp17

c_Acidobacteria_Gpl7 g Geothrix O Thermales f Thermaceae g_Desulfovibrio g_Alistipes f Rikenellaceae g_Clostridium XIVa g_Serratia

o Xanthomonadales f Sinobacteraceae

g_Hydrocarboniphaga

0 Rhodocyclaceae

f Rhodocyclaceae g Zoogloea

f_Burkholderales incertae sedls g Âquabacterium g. Hydrogenophaga g Pseudoxanthomonas g_Pseudoxanthomonas

g_Methyloversatilis

f Saprospiraceae

g Flavobacterium

gerribacterium

g_Haliscomenobacter

g_Phenylobacterium

g_Devosia

g_Methylibium

g Bacillus

f Bacillaceae 1

$\bar{g}$ Kineosporia

f_Kineosporiaceae

g Solimonas

g-Pelomonas

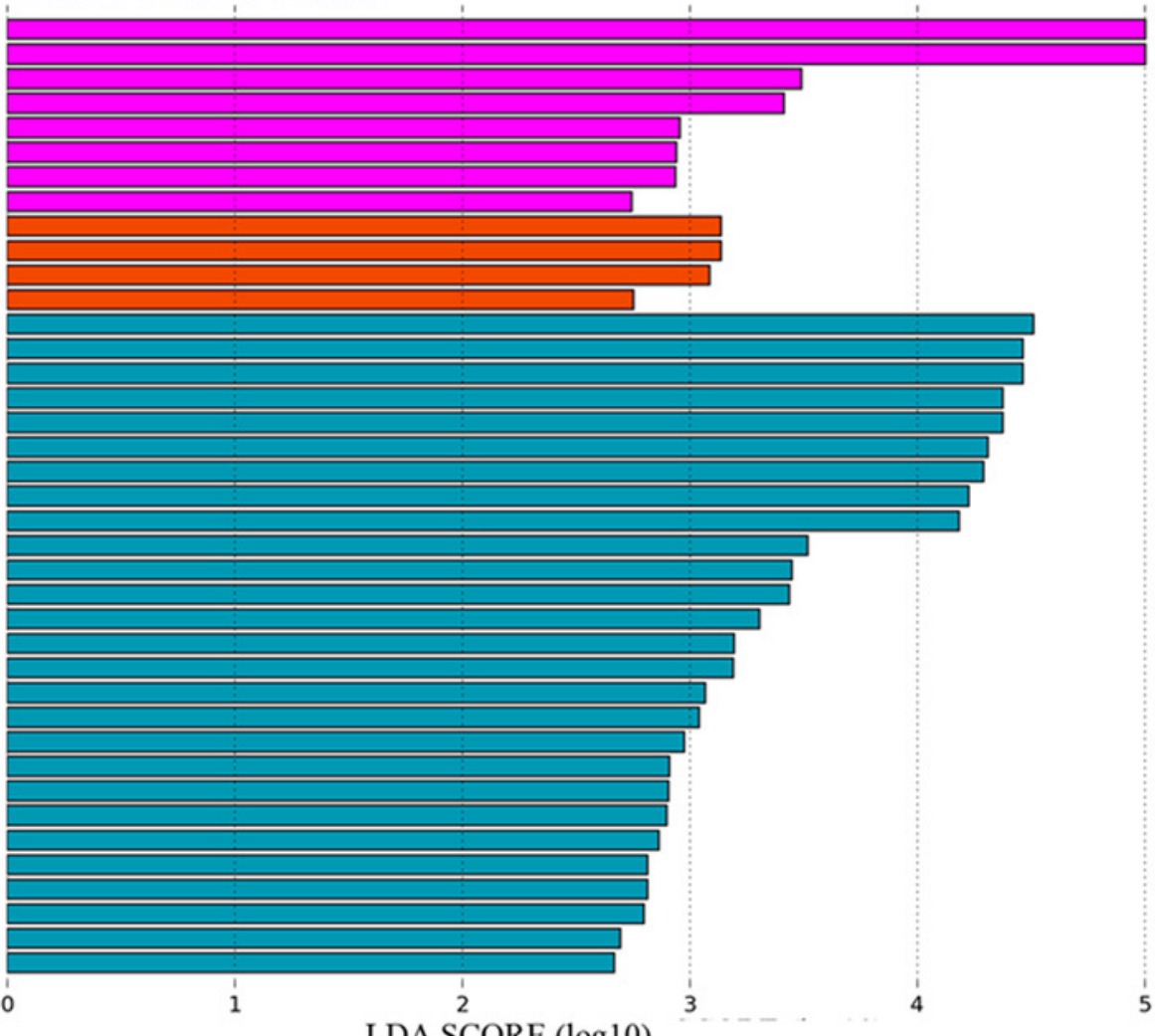

LDA SCORE $(\log 10)$ 


\section{Table 1 (on next page)}

Comparison of bacterial concentrations among the three specialties 
1

2
Table 1: Comparison of bacterial concentrations among the three specialties

\begin{tabular}{ccccc}
\hline Specialty & Sample numbers & Median(Q25,Q75) & $\boldsymbol{H}$ & $\boldsymbol{P}$ \\
\hline Periodontics & 104 & $335(116.5,1060)$ & & \\
Endodontics & 126 & $79(20,410)$ & 27.441 & $<0.01$ \\
Prosthodontics & 120 & $141(20,540)$ & & \\
\hline
\end{tabular}

3

5

6

7

8 


\section{Table 2 (on next page)}

Comparison of bacterial concentrations between samples before and after daily dental practice 
1

2

3

5
Table 2: Comparison of bacterial concentrations between samples before and after daily dental practice

\begin{tabular}{ccccc}
\hline Sampling time & Sample numbers & Median(Q25,Q75) & $\boldsymbol{Z}$ & $\boldsymbol{P}$ \\
\hline $\begin{array}{c}\text { Before daily } \\
\text { dental practice }\end{array}$ & 175 & $192(30,880)$ & & \\
$\begin{array}{c}\text { After daily } \\
\text { dental practice }\end{array}$ & 175 & $160(32,630)$ & -1.626 & 0.104 \\
\hline
\end{tabular}


Table 3(on next page)

Comparison of Alpha-diversity indices among the three specialties 
Table 3: Comparison of Alpha-diversity indices among the three specialties

2

\begin{tabular}{lcccc}
\hline Alpha-diversity & Periodontics & Endodontics & Prosthodontics & $\boldsymbol{P}$ \\
\hline Chao1 & $446.49 \pm 106.82$ & $470.13 \pm 123.29$ & $459.14 \pm 89.05$ & 0.953 \\
Observed species & $343.50 \pm 90.54$ & $383.20 \pm 121.75$ & $371.33 \pm 98.26$ & 0.731 \\
Shannon & $4.41 \pm 0.69$ & $3.77 \pm 1.75$ & $3.99 \pm 1.10$ & 0.594 \\
Simpson & $0.88 \pm 0.61$ & $0.69 \pm 0.22$ & $0.79 \pm 0.81$ & 0.159 \\
\hline
\end{tabular}

3 